\title{
MODELO CONCEITUAL PARA A SERVITIZAÇÃO DO PDP ATRAVÉS DA TRANSFERÊNCIA DE CONHECIMENTO INTERFUNCIONAL*
}

\section{CONCEPTUAL MODEL TO PDP SERVITIZATION THROUGH CROSS- FUNCTIONAL KNOWLWLEDGE TRANSFER}

\author{
Carolline Paslauski ${ }^{1, \downarrow}$, Néstor Ayala ${ }^{2}$, Débora Silveira ${ }^{3}$, Alejandro Frank $^{4}$
}

\begin{abstract}
RESUMO
No contexto de adoção da estratégia de servitização, o processo de desenvolvimento do produto (PDP) é altamente dependente da geração de novos conhecimentos para fornecer soluções de integradas de produtos e serviços. Nesse contexto, a área de suporte de produto tem o potencial de agregar ao time de PDP conhecimento sobre oportunidades, desenvolvimento e entrega de serviços. No entanto, falta orientação sobre como as empresas podem conduzir essa integração da área de suporte ao produto com outras áreas do PDP mirando na servitização da oferta. Assim, o objetivo desse artigo é propor um modelo conceitual com enfoque na transferência de conhecimento (TC) interfuncional durante o PDP para servitização. O modelo conceitual foi desenvolvido através de uma revisão da literatura sobre aspectos da TC interfuncional no PDP e da servitização. Na sequência, o modelo conceitual proposto foi confrontado com a prática por meio de seis estudos de casos em empresas servitizadas. Como resultado, o modelo conceitual proposto concentra-se em trazer a TC a partir da área de suporte de produto para os estágios iniciais da definição de problema, a fim de substituir modelos mentais tradicionais de empresas de manufatura apenas focados na oferta de produtos.
\end{abstract}

Palavras Chave: Servitização, Desenvolvimento de Produto, Transferência de Conhecimento Interfuncional.

\footnotetext{
*Versão originalmente enviada no IX Simposio Internacional de Ingeniería Industrial: Actualidad y Nuevas Tendencias 2016-Brasil e melhorada após o processo de revisão por pares da revista Ingeniería Industrial

1 Departamento de Engenharia de Produção e Transportes, Universidade Federal do Rio Grande do Sul, Porto Alegre Brasil. orcid.org/0000-0002-5616-9601.

2 Departamento de Engenharia de Produção e Transportes, Universidade Federal do Rio Grande do Sul, Porto Alegre, Brasil. orcid.org/0000-0001-8888-9227.

3 Departamento de Engenharia de Produção e Transportes, Universidade Federal do Rio Grande do Sul, Porto Alegre, Brasil. orcid.org/0000-0001-7622-6856.

4 Departamento de Engenharia de Produção e Transportes, Universidade Federal do Rio Grande do Sul, Porto Alegre, Brasil. orcid.org/0000-0001-5041-6467.
}

• Autor de correspondência: paslauski@producao.ufrgs.br 


\section{ABSTRACT}

When considered the servitization strategy, the product development process (PDP) activity is highly dependent on the generation of new knowledge to provide product-service integrated solutions. In this context, the product support area has the potential to provide to PDP knowledge about services opportunities, development and delivery. However, there is a lack of orientation on how companies can drive this integration of the product support area with other PDP areas aiming on servitization. Thus, the aim of this paper is to propose a conceptual model to foster cross-functional knowledge transfer (KT) during the PDP aiming on servitization. The conceptual model was developed by means of a literature review on cross-functional KT aspects in PDP and on servitization. In the sequence, the proposed conceptual model was confronted with the reality by means of six case studies in servitized companies. As a result, the proposed conceptual model is concentrated in bringing the KT from the product support area to the initial stages of problem definition, in order to move from outdated mental models focused on only product offers.

Keyword: Servitization, Product Development, Interfunctional Knowledge Transfer.

\section{INTRODUÇÃO}

A busca pela manutenção da competitividade de empresas de manufatura está as direcionando para adoção da estratégia de servitização (Gebauer et al., 2011). O processo de inclusão de serviços à oferta de produtos exige que empresas mudem e expandam seus conhecimentos (Leoni, 2015). Especialmente, durante o desenvolvimento da oferta, quando diferentes fontes de conhecimentos são capturadas e gerenciadas (Chirumalla, 2013).

Em empresas de manufatura, o processo de desenvolvimento de produto (PDP) tem sido abordado, principalmente, pelo aspecto da integração de conhecimento de marketing, engenharia e manufatura (Brettel et al., 2011). Assim, compreende apenas até a fase de lançamento de produtos (Huang et al., 2008), embora o lucro gerado por serviços de pósvenda em empresas de manufatura tem sido frequentemente mais elevado do que as vendas de produtos (Bundschuh \& Dezvane, 2003).

Nesse contexto, faltam na literatura estudos que incorporem a fase de pós-desenvolvimento e a área funcional responsável pelo suporte dos produtos em uso à servitização do PDP. Através da oferta de serviços como instalação, treinamento, peças de reposição e manutenção, a área organizacional de suporte ao produto possui uma posição privilegiada em relação ao cliente dentro de empresas de manufatura. Especialmente no contexto de servitização, em que a estratégia de prestação de serviços oferece fácil acesso ao feedback sobre o desempenho do produto e torna a área de suporte do produto um ator chave na captura e exploração do conhecimento que vem do cliente.

Com o objetivo de fornecer um modelo conceitual para a servitização do PDP, foi proposta a investigação, sob a lente teórica da TC interfuncional, da integração do conhecimento da área de suporte ao produto durante o PDP. Neste sentido, a oferta de serviço e o conhecimento sobre a experiência do cliente, conforme sugerem Cohen \& Levinthal (1990) na teoria da capacidade de absorção, pode ser a chave para identificação de oportunidades e de conhecimento externo no tema, capacitando a empresa a explorá-los para fins comerciais. 
Assim, a área de suporte ao produto tem a capacidade e o acesso para aquisição de novos conhecimentos sobre serviços, considerada a principal atividade de gestão de conhecimento para servitização (Leoni, 2015).

O método selecionado para atendimento do objetivo geral foi a análise de múltiplos casos, devido a sua aplicabilidade para construção de teorias baseadas em uma revisão teórica seguida de uma investigação de campo profunda (Eisenhardt \& Graebner, 2007; Yin, 2013). Este estudo visa suprir a lacuna sobre a aplicação da TC interfuncional com foco na servitização do PDP e no desenvolvimento de sistemas produto-serviço (PSS) (Leoni, 2015). Uma vez que o desenvolvimento de PSS demanda o desenvolvimento de produtos e serviços de forma integrada (Windahl \& Lakemond, 2006) e a TC interfuncional provou ser uma fonte de sucesso do PDP, pois cobre as lacunas de conhecimento que envolve a elaboração de ofertas complexas através da combinação de conhecimentos (Paslauski \& Frank, 2015), é necessário que durante o desenvolvimento de PSS ocorra a TC interfuncional. As práticas relacionadas à integração do conhecimento interfuncional no PDP podem ser estudadas em níveis estratégicos, de PDP e de times (Paslauski \& Frank, 2015). As subseções seguintes aprofundam o estudo da TC interfuncional visando a servitização do PDP sob cada uma destas abordagens.

\section{Servitização e Estratégia}

O termo servitização foi proposto por Vandermerwe \& Rada (1989) e refere-se ao processo no qual uma empresa muda a composição de sua oferta de um produto para um PSS, adicionando serviços (Baines et al., 2009). A adoção desta estratégia é justificada pela relação positiva identificada entre a adição de serviços e o aumento de valor percebido pelo mercado (Vandermerwe \& Rada, 1989).

Neste caso, a orientação declarada para as necessidades do cliente que rege esta estratégia (Vandermerwe \& Rada, 1989) é compatível com a estratégia de orientação para o mercado. Ainda, a interação com o cliente a partir da oferta de serviços abre espaço para a estratégia de orientação para a aprendizagem, fechando o ciclo de feedback entre o cliente, durante a entrega do serviço, e o P\&D da empresa (Elfving et al., 2015; Aurich et al., 2006). A combinação destas três estratégias tem potencial para viabilizar e potencializar a servitização.

Embora, em alguns casos, as estratégias orientadas para o mercado e para a aprendizagem sejam consideradas concorrentes, muitos estudos procuram explicitar a vantagem de uma combinação destas estratégias (Baker \& Sinkula, 2002). Apesar de amplamente aceita, a estratégia de orientação para o mercado é considerada insuficiente para sustentação de uma vantagem competitiva de longo prazo (Baker \& Sinkula, 2002). Neste sentido, propõese que:

Proposição 1: A servitização do PDP se beneficiaria da combinação das estratégias de orientação para o mercado e de orientação para o aprendizado.

A servitização como estratégia é caracterizada como um processo que pode evoluir em termos de orientação da oferta (Tukker, 2004) ou que pode optar diferentes tipos de serviço durante sua operacionalização (Baines \& Lightfoot, 2013). As ofertas servitizadas foram colocadas em um contínuo evolutivo entre serviço como "adicionais" até produtos como "adicionais" (Oliva \& Kallenberg, 2003) ou em um contínuo no qual os serviços evoluem de orientados para produtos, passando pela orientação para uso, até atingir a orientação para o resultado (Tukker, 2004). Quanto mais a empresa avançar nesse contínuo, ou seja, optar por uma oferta mais centrada no serviço, maior deverá ser o esforço para reconfigurar as capacidades, estruturas e base de recursos de empresas originalmente focadas na 
manufatura (Baines et al., 2009).

A orientação para o mercado pode ser definida pelo nível de influência que a assimilação de informações e conhecimento provenientes do mercado possui na formulação estratégica das empresas (Baker \& Sinkula, 2002). A orientação para a aprendizagem é caracterizada pelo nível com que as empresas proativamente confrontam seus pressupostos e suas práticas e o impacto disto na maximização do desempenho organizacional (Argyris \& Schön, 1978). Nesse contexto, a gestão do conhecimento organizacional torna-se um recurso fundamental, bem como o processo de geração de conhecimento (Baker \& Sinkula, 2002). Além disso, sugerese a institucionalização do processo de aprendizado generativo para o desenvolvimento de inovações radicais, principalmente em mercados dinâmicos (Baker \& Sinkula, 2002). Assim, sugere-se que:

Proposição 2: Diferentes níveis de servitização do PDP podem ser atingidos dependendo do nível de orientação para aprendizado desenvolvido pela empresa.

O objetivo final do processo de servitização é que uma empresa que oferta originalmente apenas produtos seja capaz de gerir uma oferta composta por produtos e serviços (Baines et al., 2009). Fica claro, assim, que a empresa precisa dispor de conhecimentos relacionados às duas parcelas da oferta. Uma forma de fazer isso é através da colaboração com fornecedores de serviços (Ayala et al., 2016; Ayala et al., 2017). No entanto, ao terceirizar completamente qualquer uma das parcelas da oferta a empresa corre o risco de perder competitividade no mercado (Porter, 2008) ou deixar que seu conhecimento sobre elas se torne obsoleto.

Na medida em que as empresas atingem um nível de criação de conhecimento mais próximo ao aprendizado generativo, a gestão de recursos deve se adaptar de forma a acompanhar esta abordagem mais dinâmica. Os recursos no contexto organizacional são definidos como ativos, capacidades, processos organizacionais, atributos da firma, informações e conhecimentos que são controlados por uma empresa e tornam possível a implementação da estratégia otimizando a eficiência e a eficácia (Barney, 1991). A visão baseada em recursos (RBV) propõe que, em ambientes dinâmicos e imprevisíveis, a vantagem competitiva pode ser facilmente alterada e os recursos que antes eram responsáveis pela diferenciação da empresa podem não ser suficientes para garantir desempenho superior no longo prazo (Helfat \& Peteraf, 2003).

Por outro lado, a teoria de capacidades dinâmicas, abordada como uma extensão construtiva da RBV (Theodosiou et al., 2012), representa um processo de modificação da base de recursos de uma empresa para obtenção de vantagem competitiva (Santos-Vijande et al., 2012). Teece et al. (1997) a definem como a capacidade de uma empresa de integrar, construir e reconfigurar experiências interna e externa para operar em ambientes dinâmicos. Recursos, capacidades dinâmicas e conhecimento estão interligados (Wright et al., 2001) e são relacionados à capacidade de redefinir continuamente a vantagem competitiva de uma empresa, tendo em vista que, em alguns contextos, a vantagem competitiva sustentável não pode ser alcançada (Fiol, 2001). Esta estratégia é uma solução para viabilizar a oferta de valor para o cliente no longo prazo em mercados dinâmicos (Santos-Vijande et al., 2012) através da constante renovação de recursos e habilidades (Wu et al., 2010). Propõe-se assim que, no que tange a gestão de recursos:

Proposição 3: A estratégia da empresa deveria migrar de RBV para capacidades dinâmicas para se tornar mais flexível e viabilizar a servitização do PDP.

$\mathrm{Na}$ busca por consistência, a abordagem de adequação à estratégia aplicada ao estudo da TC propõe o alinhamento entre os níveis estratégico e operacional das empresas (Hong 
et al., 2011). Investir na coordenação lateral entre gerentes de departamentos é possível estimular a colaboração entre áreas funcionais e a TC interfuncional (Anthony et al., 2014). O alinhamento estratégico e operacional tem sido citado como uma consequência do alinhamento das medidas de desempenho entre áreas funcionais (Hirunyawipada et al., 2010). Neste sentido, propõe-se que:

Proposição 4: O alinhamento entre os níveis estratégico e operacional é decisivo para o sucesso da servitização do PDP.

\section{Servitização do Pdp}

As principais diferenças entre o desenvolvimento de produtos nas empresas de manufatura tradicionais e de PSS estão relacionas a: (i) aumento da ênfase nos aspectos de design que têm impacto sobre as atividades de manutenção e reparação (Baines et al., 2009); (ii) processo de desenvolvimento de PSS deve ser iniciado e conduzido por um prestador de serviços, enquanto PDP é fortemente liderada pelo marketing, engenharia e manufatura (Kim et al., 2015); (iii) necessidade de uma fase de identificação de problemas para revelar requisitos do cliente anterior ao detalhamento da oferta (Aurich et al., 2006; Elfving et al., 2015); (iv) desenvolvimento de um entendimento comum sobre as necessidades dos atores envolvidos na entrega da oferta (Elfving et al., 2015); e (v) necessidade de desenvolvimento de novos canais de comunicação para apoiar a oferta de serviço durante todas as fases do ciclo de vida do produto (Aurich et al., 2006).

As ideias de serviços são geradas com base nas necessidades do cliente, novas oportunidades de serviços e condições (Moser et al., 2015). Além disso, o desenvolvimento de produto tende a ser mais centralizado em unidades de negócios, enquanto o desenvolvimento de serviços é descentralizado (Elfving et al., 2015), frequentemente co-desenvolvido com os clientes (Baines et al., 2009; Aurich et al., 2006), sendo desenvolvido e consumido simultaneamente (Aurich et al., 2006). Ainda assim, é destacada a necessidade de ter um processo de desenvolvimento mais integrado de produtos e serviços (Aurich et al., 2006; Elfving et al., 2015; Moser et al., 2015).

As macrofases do PDP, divididas em pré-desenvolvimento, desenvolvimento e pósdesenvolvimento, podem ser relacionadas respectivamente à (i) definição da oferta por marketing, (ii) desenvolvimento da oferta pela engenharia e fabricação pela manufatura e (iii) acompanhamento da oferta no mercado pelo pós-venda. Por outro lado, para o desenvolvimento de PSS, (i) os esforços estão inicialmente centrados na identificação de oportunidades e requisitos para definição do problema do cliente (Aurich et al., 2006; Schmidt et al. 2015a; Schmidt et al., 2015b), (ii) passando pelo desenvolvimento e testes da solução (Morelli, 2003; Aurich et al., 2006; Moser et al. 2015; Schmidt et al., 2015b) e (iii) incluindo o suporte na entrega da oferta e utilização do produto (Morelli 2003).

Proposição 5: Para a servitização do PDP, os objetivos das três macroetapas devem estar centradas na identificação do problema, desenvolvimento de soluções e entrega de soluções.

\section{Atores da Sertização do Pdp e a Tc Interfuncional}

A TC interfuncional tem se mostrado como fonte de sucesso do PDP em termos do desempenho do produto (Nakata \& Im, 2010) e de inovação para as empresas (Tsai et al., 2012; Baunsgaard \& Clegg, 2013). No caso do desenvolvimento de PSS, a necessidade de haver uma maior integração interfuncional para o sucesso da oferta servitizada tem sido destacada por diferentes autores (e.g. Aurich et al., 2006; Elfving et al., 2015; Moser et al. 2015).

A combinação de recursos de marketing, engenharia e produção está alinhado com a 
composição proposta por Teece (1986) como os três principais ativos de inovação. A primeira geração de inovação definida como Tecnology Push é representada pela área funcional de engenharia como P\&D, enquanto a segunda destaca a área de marketing através do Market Pull (Rothwell,1994). Da terceira geração de inovação proposta por Rothwell (1994), são fundamentais para o desenvolvimento de produtos, pelo menos, duas áreas da empresa: principalmente técnica (engenharia) e outra predominantemente comercial (marketing). A integração do conhecimento em equipes multifuncionais de PDP também destaca as interações entre marketing e manufatura (Calantone et al., 2002; Kong et al., 2015) e engenharia e manufatura (Ettlie, 1995; Jayaram \& Malhotra, 2010; Maltz, 2000) como determinantes do desempenho do PDP. Além disso, essas interações são mais bem sucedidas quando coordenadas em pares (Song et al., 1998; Troy et al., 2008) e em fases específicas do PDP (Song et al.,1998). Esta integração em pares está alinhada com a proposta para estudar o processo de transferência de conhecimento do ponto de vista de fonte e receptor (Lin et al., 2005; Frank \& Ribeiro, 2014). O sucesso da oferta servitizada está relacionada à integração de conhecimentos das áreas funcionais de marketing, engenharia, manufatura e pós-venda (Paslauski et al., 2016).

A visão estabelecida do PDP sobre as perspectivas de marketing, engenharia e manufatura tem sido questionada quando o objetivo é a oferta de PSS. A solução como serviço é gerada com base nas necessidades do cliente e nas oportunidades de mercado (Moser et al., 2015) que prevê um alinhamento entre as áreas funcionais que detém conhecimento sobre clientes e mercado.

A servitização é marcada por uma mudança estratégica com foco principal nos clientes e suas necessidades (Leoni, 2015) e a área de marketing procura novas frentes para identificar as necessidades e propor novas soluções. Neste sentido, Kim et al. (2015) propõem que o prestador de serviços deve ser responsável por fases da estratégia do planejamento e do projeto de serviço e deve participar na geração de ideias, seleção e lançamento de produtos.

Proposição 6: A TC interfuncional representa um papel importante na servitização do PDP.

\section{MATERIAIS E MÉTODOS}

As proposições apresentadas foram confrontadas com a realidade através de seis estudos de casos. Para isto, foi adotada a investigação empírica de estudo de casos múltiplos com base na coleta de dados qualitativos (Yin, 2013). Esta abordagem de pesquisa é aplicada na construção da teoria baseada na análise de campo em profundidade, quando os pesquisadores buscam compreender a ocorrência de um fenômeno (Eisenhardt \& Graebner 2007; Yin, 2013).

\section{Seleção de Casos}

De acordo com Eisenhardt \& Graebner (2007), a amostragem teórica significa que os casos são selecionados por serem adequados no esclarecimento e ampliação de relacionamentos e entre construtos. Nesse sentido, foi realizada uma análise de seis empresas multinacionais que atingiram diferentes níveis de servitização, apresentadas na Tabela 1. Buscou-se assim, produzir resultados contrastantes que permitissem produzir uma visão ampla do problema com intuito de favorecer a generalização dos resultados (Ceschin, 2013; Yin, 2013). 
Tabela 1. Descrição dos casos analisados

\begin{tabular}{lll}
\hline \multicolumn{1}{c}{ DESCRIÇÃO } & \multicolumn{1}{c}{ ENTREVISTADO } \\
\hline A $\begin{array}{ll}\text { Multinacional alemã do setor de } \\
\text { equipamentos odontológico, com mais } \\
\text { de } 1.000 \text { funcionários e presente em } \\
\text { mais de } 36 \text { países. }\end{array}$ & $\begin{array}{l}\text { CEO da América Latina, com 12 anos de } \\
\text { empresa e o principal responsável pela } \\
\text { estruturação dos negócios de servitização } \\
\text { na região. }\end{array}$ \\
\hline $\begin{array}{l}\text { Multinacional norte-americana de TI } \\
\text { (hardware e serviços), com mais de } \\
100.000 \text { funcionários em todo o mundo. }\end{array}$ & $\begin{array}{l}\text { Gerente da área de serviços e integrante } \\
\text { do time de PDP local. }\end{array}$ \\
\hline Cultinacional sueca do setor de & $\begin{array}{l}\text { Gerente de projetos com 10 anos de } \\
\text { telecompresa. Já trabalhou em filiais latino- } \\
100.000 \text { funcionários operando em mais } \\
\text { de } 180 \text { países. }\end{array}$ & $\begin{array}{l}\text { americanas da empresa, atuando no } \\
\text { processo de servitização na região. }\end{array}$ \\
\hline $\begin{array}{l}\text { Multinacional suiça do setor de } \\
\text { automação e energia com mais de } \\
\text { 140.000 funcionários e presente em } \\
\text { mais de 100 países. }\end{array}$ & $\begin{array}{l}\text { Gerente de projetos com 12 anos de } \\
\text { empresa participou do processo de } \\
\text { servitização da empresa na Europa e } \\
\text { fornece consultoria interna de processos } \\
\text { na mesma região. }\end{array}$ \\
\hline $\begin{array}{l}\text { Multinacional francesa do setor de } \\
\text { automação e energia com mais de } \\
\text { 150.000 funcionários em mais de 100 } \\
\text { países. }\end{array}$ & $\begin{array}{l}\text { Gerente da área de serviços dedicado ao } \\
\text { desenvolvimento de soluções (produto- } \\
\text { serviço). }\end{array}$ \\
\hline $\begin{array}{l}\text { Multinacional norte-americana do setor } \\
\text { de processo e controle de movimento } \\
\text { com aproximadamente } 8.000 \\
\text { funcionários e filiais em 49 países. }\end{array}$ & $\begin{array}{l}\text { Gerente de cadeia de suprimentos com } \\
15 \text { anos de empresa, encarregado das } \\
\text { relações entre as atividades industriais e } \\
\text { os serviços. }\end{array}$ \\
\hline
\end{tabular}

\section{Coleta de Dados}

O processo de coleta de dados foi realizado entre março e agosto de 2015 através de duas fontes de informação, com intuito de ampliar a confiabilidade da análise (Yin, 2013). Como fonte primária, foram utilizadas entrevistas semiestruturadas com gerentes que participaram diretamente do processo de servitização das empresas e que possuem uma relação próxima ao desenvolvimento de produtos e/ou serviços. As entrevistas foram gravadas em áudio e posteriormente transcritas. Com fonte secundária, foram analisados documentos das empresas (relatórios de negócios e apresentações de slides internas), informações da página institucional e artigos científicos que continham estudos de caso realizados nas mesmas empresas.

\section{Análise dos Dados}

Foi adotada uma abordagem dedutiva, para explorar se os elementos propostos no modelo conceitual poderiam ser reconhecidos nos casos, seguida de uma abordagem indutiva para proposição de novos elementos relevantes à servitização do PDP (Van de Ven, 2007). Cada caso foi analisado individualmente, para identificação de fatores e comportamentos isolados, e através do cruzamento de casos, para reconhecimento de semelhanças, contrastes e padrões. Por fim, os resultados foram integrados aos achados da literatura e produziu-se um modelo conceitual para a servitização do PDP. 


\section{RESULTADOS E DISCUSSÕES O PAPEL DA ESTRATÉGIA NA SERVITIZAÇÃO DO PDP}

\section{Proposição 1}

Há uma tendência de orientação para mercado nas motivações apresentadas pelas empresas para servitização: necessidade de sobrevivência no mercado (empresa A e F) ou entrada em um novo mercado (empresa A), a busca por diferenciação (empresa A e C) e aumento do lucro (empresa E). Complementarmente, a orientação para aprendizagem está relacionada com a customização da oferta (empresa A e E) e o feedback sobre o uso do produto e a identificação de oportunidades de novos produtos/serviços (empresa A).

As motivações mencionadas estão alinhadas com as principais vantagens relacionadas à inclusão de serviços na oferta de produtos propostas na literatura. No que tange a estratégia de orientação para mercado, busca-se garantir o benefício esperado do produto, ampliar os benefícios dos produtos por meio de ofertas complementares e influenciar o ciclo de vida do produto em termos de disponibilidade, desempenho, tempo de vida e eco eficiência (Aurich et al., 2006). No caso da orientação para aprendizagem, busca-se fechar o ciclo de feedback do produto através da prestação de serviços e da interação com o cliente (Aurich et al., 2006; Elfving et al. 2015) visando a melhoria continua de produtos e serviços (Aurich et al., 2006; Muto et al. 2015). Estas declarações apoiam a primeira proposição de combinar a estratégia de servitização com as estratégias de orientação para mercado e para aprendizagem.

\section{Proposição 2}

Ainda, apesar de ter um percentual da receita nas empresas igual ou superior a $25 \%$, os serviços têm relevâncias diferentes para as unidades das empresas que desenvolvem produtos e tecnologia (empresas C, D e E) e para aquelas que simplesmente montam, adaptam e/ou vendem produtos desenvolvidos por uma matriz (empresas A, B e F) (Tabela 2). O grupo composto pelas empresas $A, B$ e $F$ está mais centrada nos serviços de entrega e pós-venda, buscando ampliar a confiança do cliente no produto através de ações paralelas ao PDP e treinar o cliente na utilização do produto (ao invés de repensar o produto para se adequar ao cliente). O grupo composto pelas empresas C, D e E visa, principalmente, reutilizar o conhecimento de engenharia sobre produtos na oferta de consultoria e no desenvolvimento de serviços puros ou softwares, devido a sua participação no desenvolvimento conjunto de produtos e serviços.

Tabela 2. Serviços disponibilizados pelas empresas estudadas

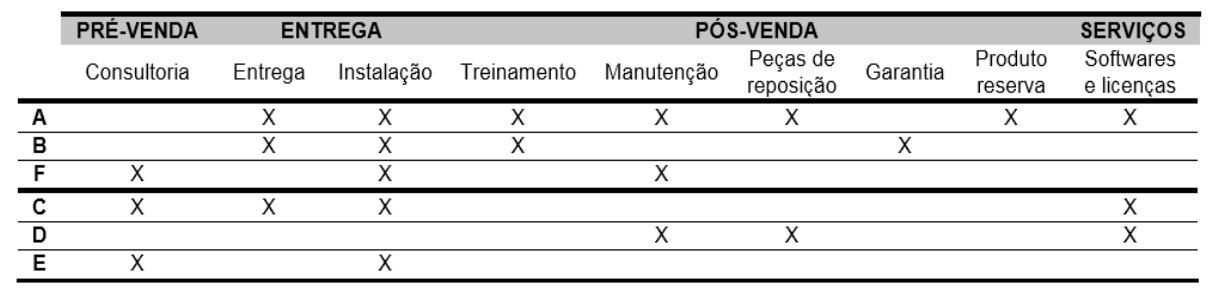

Estas descobertas justificam a segunda proposição, no que se refere à influência do nível em que os modelos mentais organizacionais são contestados na orientação da servitização alcançada. Enquanto as empresas A, B e F prestam serviços orientadas principalmente para produtos (entrega, instalação, treinamento, manutenção, peças de reposição, garantia e produtos de reserva), as empresas C, D e prestam serviços orientados ao uso (softwares e licenças) e resultados (consultoria), de acordo com a classificação de Tukker (2004). No entanto, a partir dos estudos de caso percebeu-se que a prediposição ao questionamento de 
modelos mentais estabelecidos é uma consequência, e não um determinante, da estratégia individual da unidade da empresa.

\section{Proposição 3}

Por outro lado, sugerindo que a mesma empresa pode trabalhar com ofertas posicionadas em diferentes níveis de servitização, foram identificados (i) a prestação de serviço de entrega e instalação das empresas $\mathrm{C}$ e $\mathrm{E}$ e A (ii) a oferta de software pela empresa A. Ao declarar que "a linha de produtos leves tem menos serviços porque eles são peças padrão", a empresa E chamou a atenção para o fato de que a mesma empresa pode trabalhar em diferentes níveis de servitização e com orientações diferentes de serviços. Esta constatação reforça a necessidade de uma estratégia de capacidades dinâmicas para apoiar a estratégia de servitização, apresentada na proposição 3.

\section{Proposição 4}

Por fim, as limitações relacionadas com a adequação da estratégia também foram mencionadas, como a dificuldade de precificação e cobrança por serviços quando a gestão financeira organizacional é elaborada para produtos (empresas A e B) e de ver reconhecidas as atividades relacionadas à oferta de serviços, quando os indicadores de desempenho não a favorecem (empresas D e E). Essas declarações reforçam a necessidade de aplicação de práticas para garantir a adequação estratégica, tal como sugerido na quarta proposição. São práticas comuns destacadas na literatura de TC interfuncional no PDP a coordenação lateral entre áreas funcionais (Anthony et al., 2014) e o alinhamento das medidas de desempenho destas (Hirunyawipada et al., 2010).

\section{O Papel da Tc Interfuncional na Servitização do PDP}

\section{Proposição 5}

A necessidade de reunir diferentes especialidades na fase inicial do desenvolvimento da oferta com o objetivo de identificar as demandas dos clientes foi destacada de diferentes formas pelas empresas estudadas. Na empresa $\mathrm{C}$, somente na fase de entrega percebiase que existia uma "grande lacuna entre o que foi negociado com o cliente pela equipe de vendas e o que a equipe de engenharia poderia entregar". Como solução para este mesmo problema, a empresa $\mathrm{F}$ determinou que representantes da área de engenharia deveriam participar das fases iniciais do PDP, o que resultou em redução da insatisfação dos clientes. Em outros casos, a necessidade de participação da área de suporte ao produto foi percebida quando o produto já havia sido entregue para o cliente. Por exemplo, a necessidade de repensar a interface do software surgiu na empresa $A$ a partir da alta demanda sobre a área de suporte ao produto para esclarecer dúvidas e resolver problemas técnicos. Nas empresas $\mathrm{B}$ e $\mathrm{E}$, as lacunas resultantes do envolvimento tardio da área de suporte ao produto no processo são atenuadas pela coordenação do desenvolvimento por um agente neutro: "Um generalista, que atua como ponto de contato com o cliente, e é uma pessoa que fala sobre tudo. Ele identifica oportunidades e é responsável pela contratação de peritos e engenheiros para projetar, por exemplo, o hardware e onde minha equipe vem, os serviços."

No entanto, ainda há uma falta de integração entre o desenvolvimento de produtos e serviços. No caso da empresa $C$ "há uma equipe de desenvolvimento de produto e outra equipe para planejar a entrega" e, na empresa D, o PDP está dividido em desenvolvimento de produto e desenvolvimento do serviço de suporte ao produto de forma paralela. Mas a empresa $D$ reconhece a necessidade de a "engenharia de serviços visar a melhoria do sistema do cliente e não apenas no desenvolvimento de produtos e vendas". Mesmo declarando que as parcelas de produtos e serviço da oferta não são pensadas em conjunto, existe um entendimento do impacto positivo que esta atitude traria e um esforço em incluir o cliente 
nas etapas de identificação de necessidades, como apresentado na proposição 5.

\section{Proposição 6}

Sobre a TC interfuncional, as considerações discorrem sobre a falta reconhecida e o impacto negativo disto no desenvolvimento de PSS. A empresa A, ao tentar desenvolver um software internamente com a equipe de engenharia, falou que "a comunicação foi toda errada, porque o cara estava na área de TI, e ele não se importava com quem ele estava falando - se era com o cliente ou quem era... eles não sabiam como lidar, porque as línguas eram diferentes". O representante do suporte ao produto na empresa B disse que eles tinham "realmente uma pequena influencia [no desenvolvimento do produto ou serviço], regionalmente". E a empresa $D$ declarou que "não possui um consenso da gestão para incentivar o intercâmbio de conhecimento entre as áreas", que "ainda há barreiras culturais entre os departamentos para impedi-lo" e reclama que "os engenheiros envolvidos no processo (PDP) tem pouca disponibilidade para apoiar a equipe de serviço".

A centralização da atividade de P\&D, para estar perto das fontes de desenvolvimento tecnológico, e a descentralização das atividades de pós-venda, com o intuito de estar mais próximo dos clientes, faz com que muitas multinacionais mantenham a engenharia em países desenvolvidos e o setor de pós-venda em países em desenvolvimento, como foi o caso relatado por todas as empresas entrevistadas. Para superar este gap, unidades de empresas multinacionais atuando em mercados de economia emergente se valem da adição de serviços na oferta de produtos com intuito de flexibilizar a oferta e adaptá-la à um mercado que não era o principal foco do PDP (Paslauski et al., 2017).

Por outro lado, a participação da área de suporte ao produto na fase de pós-desenvolvimento reforçou o papel desta área no fechamento do ciclo de aprendizagem organizacional, onde a empresa A declara que a prestação de serviços tem estimulado o desenvolvimento de novos produtos, a melhoria dos produtos atuais e a venda de produtos atuais para novos clientes. Assim, segue-se reforçando a proposição 6 sobre a importância da TC interfuncional, que pode ser alcançada através da integração em pares de áreas funcionais, cujo conhecimento necessário depende da etapa do PDP (Kong et al., 2015).

Combinando os resultados encontrados na literatura e nos estudos de caso, foi proposto o modelo da Figura 1 para empresas que buscam orientação para servitização do PDP.

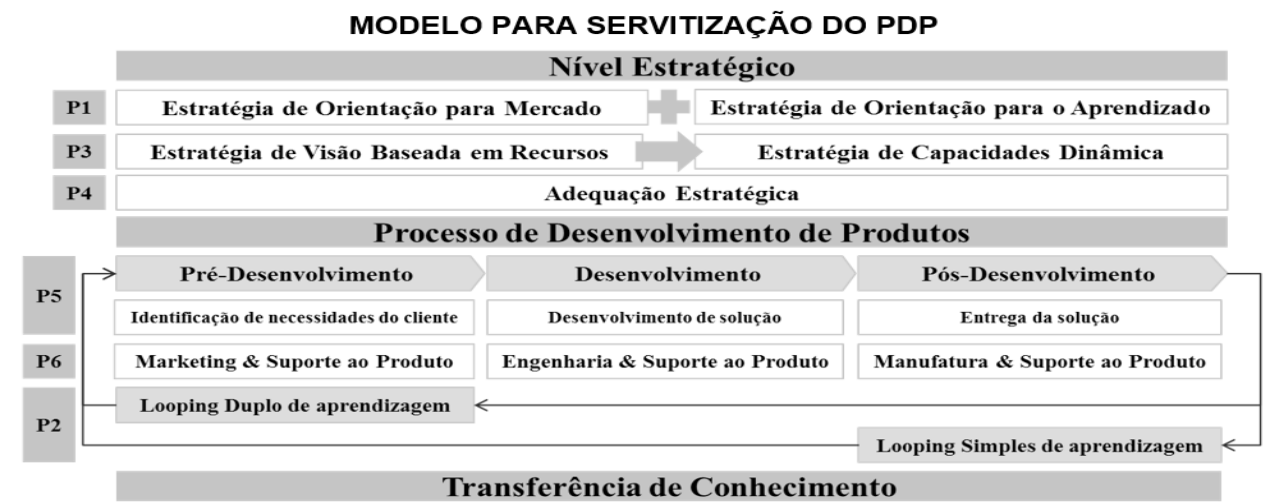

Figura 1. Modelo para servitização do PDP 
Este modelo é organizado com base nos três níveis discutidos na estratégia de fundo teórico, processo de desenvolvimento de produto e integração do processo de conhecimento. No nível da estratégia, a orientação do mercado é representada por um forte estágio de definição do problema, sem preocupação com a solução que propõe nesse ponto, enquanto a orientação para a aprendizagem é representada através da presença da área de pós-venda em diferentes estágios do PDP, impactando de formas diferentes no ciclo de aprendizagem organizacional. A viabilidade dessa integração ocorre, em um primeiro momento, apoiada por uma estratégia de visão baseada em recursos e, à medida que se atinja um alto nível de participação do pós-venda na etapa de pré-desenvolvimento, evoluirá para uma estratégia de capacidade dinâmica. $\mathrm{O}$ ajuste estratégico é proposto para ser alcançado através da distribuição igual de poder entre áreas funcionais e indicadores complementares interdepartamentais. Além das interações funcionais tradicionais entre as três áreas funcionais: Marketing, Engenharia e Manufatura, para o desenvolvimento do PSS, identificamos a necessidade de integrar: (i) engenharia e pós-venda nas fases pré-desenvolvimento e desenvolvimento (Kim et al., 2015) ; (li) marketing e pós-venda na fase de pré-desenvolvimento, como proposto para a independência da visão de produto com a qual a atividade de marketing é abordada (Shostack 1977), sob a forma de marketing de produtos e serviços; e (iii) pós-venda e manufatura durante a fase de pós-desenvolvimento com o objetivo de fechar o ciclo de feedback entre a entrega de serviços e a interação do cliente com o P\&D da empresa (Aurich et al., 2006; Elfving, 2015).

Para a integração do conhecimento, a proposta teórica de Argyris e Schön (1978) de ciclos simples e duplo de aprendizagem foi aplicada através da integração da área de pós-venda na fase de pós-desenvolvimento e na fase de pré-desenvolvimento, respectivamente. $O$ aprendizado de ciclo único envolve aprendizagem adaptativa para a detecção e correção de erros propostos na teoria explícita em uso sem, no entanto, a reavaliação de modelos mentais que o suportam (Baker \& Sinkula, 2002). Isto é proposto para ser alcançado pela área de suporte do produto na fase pós-desenvolvimento e pela exploração de seu potencial de integração intertemporal de conhecimento que pode ser aplicado na atividade de melhoria contínua (Aurich et al., 2006). Isso deve resultar em serviços orientados para o produto (Tukker, 2004) e deve ser apoiado por uma estratégia baseada em recursos, uma vez que possibilite a identificação dos novos recursos necessários para adaptação (Helfat \& Peteraf 2003, Santos-Vijande et al., 2012). No entanto, para obter mais serviços orientados para o uso e resultados (Tukker, 2004), a empresa deve ser capaz de expor modelos mentais limitados e incorrer no ciclo duplo de aprendizagem (Argyris, 1976). Assim, a empresa questiona a oferta de produtos como uma solução para necessidades complexas e repensa sua estratégia para uma abordagem de capacidade dinâmica na qual ela pode desenvolver produtos, serviços ou PSS visando a satisfação das necessidades do cliente.

\section{CONCLUSÕES}

O modelo conceitual proposto de utilização da lente teórica da TC interfuncional para servitização do PDP está centrado no envolvimento da área de suporte ao produto a partir dos estágios iniciais da definição do problema, de forma a reforçar seu papel de gestão do ciclo de vida do produto e de aprendizagem contínua. As proposições de orientação para o mercado, orientação para aprendizagem, capacidades dinâmicas, ajuste estratégico e participação da área de suporte do produto no PDP foram reforçados nos estudos de caso. No entanto, a amostra de empresas só nos permitiu verificar a hipótese de aprendizagem do looping simples, uma vez que seria necessário entrevistar empresas em um nível mais 
avançado de servitização e com um nível mais elevado de integração multidisciplinar para compreensão do looping duplo. Este estudo fornece uma ponte entre prática e teoria nos níveis estratégico e processual da servitização do PDP. Para estudos futuros propõe-se a verificação quantitativa do modelo, especialmente no que tange o impacto das interações entre áreas propostas no sucesso do PSS.

\section{AGRADECIMENTOS}

Os autores agradecem à Coordenação de Aperfeiçoamento Pessoal de Nível Superior (Capes) e ao Conselho Nacional de Desenvolvimento Científico e Tecnológico (CNPq) do Ministério da Educação (MEC), Governo Federal do Brasil, pela concessão de bolsas de mestrado e doutorado que viabilizaram esta pesquisa.

\section{REFERÊNCIAS}

ANTHONY, E.L., GREEN, S.G and MCCOMB, S.A. Crossing functions above the crossfunctional project team: The value of lateral coordination among functional department heads. Journal of Engineering and Technology Management, 2014, 31, 141-158.

ARGYRIS, C and SCHÖN, D.A. Organizational learning. Reading, Mass.: Addison-Wesley Pub. Co., 1978. Addison-Wesley series on organization development. ISBN 0-201-00174-8

ARGYRIS, C. Single-loop and double-loop models in research on decision making. Administrative science quarterly, 1976, 363-375.

AURICH, J.C., FUCHS, C and WAGENKNECHT, C. Life cycle oriented design of technical Product-Service Systems. Journal of Cleaner Production, 2006, 14(17), 1480- 1494.

AYALA, N.F., PASLAUSKI, C. A., RIBEIRO, J. L.D and FRANK, A.G. An analysis of buyersupplier integration for servitization strategies. Procedia CIRP, 2016, 47, 388-393.

AYALA, N. F., PASLAUSKI, C. A., GHEZZI, A and FRANK, A.G. Knowledge sharing dynamics in service suppliers' involvement for servitization of manufacturing companies. International Journal of Production Economics, 2017, 193, 538-553.

BAINES, T., LIGHTFOOT, H., PEPPARD, J., JOHNSON, M., TIWARI, A., SHEHAB, E and SWINK, M. Towards an operations strategy for product-centric servitization. International Journal of Operations \& Production Management, 2009, 29(5), 494-519.

BAINES, T., W and LIGHTFOOT, H. Servitization of the manufacturing firm: Exploring the operations practices and technologies that deliver advanced services. International Journal of Operations \& Production Management, 2013, 34(1), 2-35.

BAKER, W. E and SINKULA, J. M. Market orientation, learning orientation and product innovation: delving into the organization's black box. Journal of market-focused management, 2002, 5(1), 5-23. 
BARNEY, J. Firm resources and sustained competitive advantage. Journal of management, 1991, 17(1), 99-120.

BAUNSGAARD, V. V and CLEGG, S. 'Walls or boxes': The effects of professional identity, power and rationality on strategies for cross-functional integration. Organization Studies, 2013, 34(9), 1299-1325.

BRETTEL, M., HEINEMANN, F., E. A and NEUBAUER, S. Cross-functional integration of R\&D, marketing, and manufacturing in radical and incremental product innovations and its effects on project effectiveness and efficiency. Journal of Product Innovation Management, 2011, 28(2), 251-269.

BUNDSCHUH, R. G and DEZVANE, T. M. How to make after-sales services pay off. McKinsey Quarterly, 2003, (4), 116-127.

CALANTONE, R., DRÖGE, C and VICKERY, S. Investigating the manufacturing-marketing interface in new product development: does context affect the strength of relationships? Journal of Operations Management, 2002, 20(3), 273-287.

CESCHIN, F. Critical factors for implementing and diffusing sustainable product-Service systems: insights from innovation studies and companies' experiences. Journal of Cleaner Production, 2013, 45, 74-88.

CHIRUMALLA, K. Managing knowledge for product-service system innovation: the role of Web 2.0 technologies. Research-Technology Management, 2013, 56(2), 45-53.

COHEN, W. M and LEVINTHAL, D. A. Absorptive capacity: A new perspective on learning and innovation. Administrative science quarterly, 1990, 128-152.

EISENHARDT, K. M and GRAEBNER, M. E. Theory building from cases: Opportunities and challenges. Academy of management journal, 2007, 50(1), 25.

ELFVING, S. W., LINDAHL, M and SUNDIN, E. Ericsson-The History from Product to Solution Provider and Challenges and Opportunities in an Evolving Environment. Procedia CIRP, 2015, 30, 239-244.

ETTLIE, J.E. Product-process development integration in manufacturing. Management Science, 1995, 41(7), 1224-1237.

FIOL, C. M. Revisiting an identity-based view of sustainable competitive advantage. Journal of Management, 2001, 27(6), 691-699.

FRANK, A. G and RIBEIRO, J. L. D. An integrative model for knowledge transfer between new product development project teams. Knowledge Management Research \& Practice, 2014, 12(2), 215-225.

GEBAUER, H., GUSTAFSSON, A and WITELL, L. Competitive advantage through service differentiation by manufacturing companies. Journal of Business Research, 2011, 64(12), 1270-1280.

HELFAT, C. E and PETERAF, M. A. The dynamic resource based view: Capability lifecycles. Strategic management journal, 2003, 24(10), 997-1010. 
HIRUNYAWIPADA, T., BEYERLEIN, M and BLANKSON, C. Cross-functional integration as a knowledge transformation mechanism: Implications for new product development. Industrial Marketing Management, 2010, 39(4), 650-660.

HONG, P., DOLL, W. J., REVILLA, E and NAHM, A.Y. Knowledge sharing and strategic fit in integrated product development projects: An empirical study. International Journal of Production Economics, 2011, 132(2), 186-196.

HUANG, C. M., CHANG, H. C and HENDERSON, S. Knowledge transfer barriers between research and development and marketing groups within Taiwanese small and medium sized enterprise high technology new product development teams. Human Factors and Ergonomics in Manufacturing \& Service Industries, 2008, 18(6), 621-657.

JAYARAM, J and MALHOTRA, M.K. The differential and contingent impact of concurrency on new product development project performance: A holistic examination. Decision Sciences, 2010, 41(1), 147-196.

KIM, S., SON, C., YOON, B and PARK, Y. Development of an Innovation Model Based on a Service-Oriented Product Service System (PSS). Sustainability, 2015, 7(11), 14427-14449.

KONG, T., LI, G., FENG, T and SUN, L. Effects of marketing-manufacturing integration across stages of new product development on performance. International Journal of Production Research, 2015, 53(8), 2269-2284.

LEONI, L. Adding service means adding knowledge: an inductive single-case study. Business Process Management Journal, 2015, 21(3), 610-627.

LIN, L., GENG, X and WHINSTON, A. B. A sender-receiver framework for knowledge transfer. Mis quarterly, 2005, 197-219.

MALTZ, E. Is all communication created equal?: An investigation into the effects of communication mode on perceived information quality. Journal of Product Innovation Management, 2000, 17(2), 110-127.

MORELLI, N. Product-service systems, a perspective shift for designers: A case study: the design of a telecentre. Design Studies, 2003, 24(1), 73-99.

MOSER, U., MAISENBACHER, S., KASPEREK, D and MAURER, M. Definition of an Approach for the Development of Product-Service Systems. Procedia CIRP, 2015, 30, 18- 23.

MUTO, K., KIMITA, K and SHIMOMURA, Y. A Guideline for Product-Service- Systems Design Process. Procedia CIRP, 2015, 30, 60-65.

NAKATA, C. and IM, S. Spurring Cross- Functional Integration for Higher New Product Performance: A Group Effectiveness Perspective. Journal of Product Innovation Management, 2010, 27(4), 554-571.

PASLAUSKI, C. A and FRANK, A. G. Perspectiva para estudo da Transferência de Conhecimento em Times Multidisciplinares de Desenvolvimento de Produtos. In: Congresso Brasileiro de Gestão da Inovação e Desenvolvimento de Produtos, 10, 2015, Itajubá/MG, Anais. 
PASLAUSKI, C. A., AYALA, N. F., TORTORELLA, G. L and FRANK, A. G. The Last Border for Servitization. Procedia CIRP, 2016, 47, 394-399.

PASLAUSKI, C. A., ALENCASTRO, C. G., AYALA, N. F., GAIARDELLI, P., PEZZOTTA, G and FRANK, A. G. Services Extending Products: a comparative analysis in emerging and developed countries. Procedia CIRP, 2017, 64, 127-132.

PORTER, M. E. The five competitive forces that shape strategy. Harvard business review, 2008, 86(1), 25-40.

ROTHWELL, R. Towards the fifth-generation innovation process. International marketing review, 1994, 11(1), 7-31.

SANTOS-VIJANDE, M. L., LÓPEZ-SÁNCHEZ, J. Á and TRESPALACIOS, J. A. How organizational learning affects a firm's flexibility, competitive strategy, and performance. Journal of Business Research, 2012, 65(8), 1079-1089.

SCHMIDT, D. M., MALASCHEWSKI, O., FLUHR, D and MÖRTL, M. Customer-oriented Framework for Product-service Systems. Procedia CIRP, 2015a, 30, 287-292.

SCHMIDT, D. M., MALASCHEWSKI, O and MÖRTL, M. Decision-making process for Product Planning of Product-Service Systems. Procedia CIRP, 2015b, 30, 468-473.

SHOSTACK, G. L. Breaking free from product marketing. The Journal of Marketing, 1977, 73-80.

SONG, X. M., THIEME, R. J and XIE, J. The impact of cross-functional joint involvement across product development stages: an exploratory study. Journal of product innovation management, 1998, 15(4), 289-303.

TEECE, D. J., PISANO, G and SHUEN, A. Dynamic capabilities and strategic management. Strategic management journal, 1997, 18(7), 509-533.

TEECE, D. J. Profiting from technological innovation: Implications for integration, collaboration, licensing and public policy. Research policy, 1986, 15(6), 285-305.

THEODOSIOU, M., KEHAGIAS, J and KATSIKEA, E. Strategic orientations, marketing capabilities and firm performance: An empirical investigation in the context of frontline managers in service organizations. Industrial Marketing Management, 2012, 41(7), 10581070.

TROY, L. C., HIRUNYAWIPADA, T and PASWAN, A. K. Cross-functional integration and new product success: an empirical investigation of the findings. Journal of Marketing, 2008, 72(6), 132-146.

TSAI, K. H., HSU, T. T and FANG, W. Relinking Cross-functional Collaboration, Knowledge Integration Mechanisms, and Product Innovation Performance: a Moderated Mediation Model. Canadian Journal of Administrative Sciences/Revue Canadienne des Sciences de I'Administration, 2012, 29(1), 25-39.

TUKKER, A. Eight types of product-service system: eight ways to sustainability? Experiences 
from SusProNet. Business strategy and the environment, 2004, 13(4), 246-260.

VAN DE VEN, A. H.. Engaged scholarship: a guide for organizational and social research: a guide for organizational and social research. Reading, Mass.: Oxford University Press on Demand, 2007. ISBN: 978-0-19-922630-6

VANDERMERWE, S and RADA, J. Servitization of business: adding value by adding services. European Management Journal, 1989, 6(4), 314-324.

WINDAHL, C and LAKEMOND, N. Developing integrated solutions: The importance of relationships within the network. Industrial Marketing Management, 2006, 35(7), 806-818.

WRIGHT, P. M., DUNFORD, B. B and SNELL, S. A. Human resources and the resource based view of the firm. Journal of management, 2001, 27(6), 701-721.

WU, S. J., MELNYK, S. A and FLYNN, B. B. Operational capabilities: The secret ingredient. Decision Sciences, 2010, 41(4), 721-754.

YIN, R. K. Case study research: Design and methods. Reading, Mass.: Sage publications, 2013, ISBN 978-1-45-224256-9. 